\title{
Performance of Mobile Telecommunications Network With Overlapping Location Area Configuration
}

\author{
Shun-Ren Yang, Yung-Chun Lin, and Yi-Bing Lin, Fellow, IEEE
}

\begin{abstract}
In a mobile telecommunications network, the location area (LA) of a mobile station (MS) is tracked by an LA update (LAU) mechanism. To reduce the LAU traffic caused by the ping-pong effect, the overlapping $L A$ concept is introduced. In the overlapping LA configuration, an LA selection policy is required to select the new LA at an LAU when the MS enters a new cell covered by multiple LAs. This paper describes four LA selection policies and proposes an analytic model to study the performance of these LA selection policies. Our study provides guidelines to determine an appropriate degree of overlapping among the LAs.
\end{abstract}

Index Terms-Location area (LA), location update, mobility management, overlapping LA, ping-pong effect.

\section{INTRODUCTION}

$\mathbf{I}$ N A MOBILE telecommunications network, the cells (the coverage areas of base stations) are partitioned into groups. These groups are referred to as the location areas (LAs; in GSM [7] and in the CS domain in GPRS/UMTS [8]), the routing areas (in the PS domain in GPRS/UMTS), the paging groups (in WiMax [6]), and so on. Without loss of generality, we use the term LA throughout this paper. The LA of a mobile station (MS) is tracked by the network. Whenever the MS moves from one LA to another LA, the MS issues an LA update (LAU) request to inform the network that it has entered the new LA. This way, the network keeps track of the LA in which the MS resides.

A typical LA layout is shown in Fig. 1(a), where every cell is covered by exactly one LA. Recently, the overlapping LA

Manuscript received July 1, 2006; revised December 2, 2006, February 9, 2007, and May 22, 2007. This work was supported in part by the National Science Council of Taiwan under Contracts NSC 94-2752-E-007-003-PAE, NSC 94-2213-E-007-072, NSC 94-2219-E-009-024, NSC 95-2752-E-009-005PAE, NSC 95-2218-E-009-201-MY3, NSC 94-2219-E-009-001, and NSC 94-2219-E-009-024, by the NTP SIP-based B3G project under Grant NSC 95-2219-E-009-010, by the NTP IMS Integration Project under Grant NSC 952219-E-009-019, by Intel, by Chung Hwa Telecom, by IIS/Academia Sinica, by the ITRI/NCTU Joint Research Center, and by MoE ATU. The review of this paper was coordinated by Dr. E. Hossain.

S.-R. Yang is with the Department of Computer Science and Institute of Communications Engineering, National Tsing Hua University, Hsinchu 300, Taiwan, R.O.C. (e-mail: sryang@cs.nthu.edu.tw).

Y.-C. Lin is with the Department of Computer Science, National Chiao Tung University, Hsinchu 30010, Taiwan, R.O.C. (e-mail: yjlin@ csie.nctu.edu.tw).

Y.-B. Lin is with the Department of Computer Science, National Chiao Tung University, Hsinchu 30010, Taiwan, R.O.C. and also with the Institute of Information Science, Academia Sinica, Taipei 115, Taiwan, R.O.C. (e-mail: liny@csie.nctu.edu.tw).

Color versions of one or more of the figures in this paper are available online at http://ieeexplore.ieee.org.

Digital Object Identifier 10.1109/TVT.2007.905423 concept [where a cell is covered by multiple LAs; see the gray areas in Fig. 1(b)] has been proposed to avoid the ping-pong effect [1]-[5], [9]. Suppose that an MS resides in cell $a$ of LA 1, as shown in Fig. 1. In the nonoverlapping LA configuration [Fig. 1(a)], when the MS moves to cell $b$ in LA 2, the MS performs an LAU to register to LA 2. If the MS moves to cell $c$ or back to cell $a$ in LA 1 again, the MS performs another LAU to register to LA 1 . If the MS repeatedly moves back and forth between LA 1 and LA 2, many LAUs are performed. This phenomenon is called the ping-pong effect. In the overlapping LA configuration, when the MS moves from cell $a$ (in LA 1) to cell $b$ (in LA 2), an LAU is executed as in the nonoverlapping LA configuration. If the MS moves to cell $c$ or back to cell $a$, it still resides in LA 2, and no LAU is performed. Therefore, the ping-pong effect is mitigated.

Several studies [1], [2], [5] proposed analytic models to study the performance of the mobile telecommunications network with overlapping LAs. These studies assumed that at each movement, the MS moves to any of its neighboring cells with the same probability. In [5], the LA consists of odd numbers of cells. In [1] and [2], an example is provided, and no close-form solution was derived to evaluate the performance. Moreover, each of the previous studies investigated one LA selection algorithm. In this paper, we propose an analytic model with a 1-D overlapping LA configuration to investigate the performance for four LA selection policies where the MS can move to each of its neighboring cells with different probabilities.

This paper is organized as follows. Section II introduces the system model and four overlapping LA policies. Section III proposes the analytic models for these policies. Section IV quantitatively compares the four studied policies. Then, Section $\mathrm{V}$ provides guidelines to determine the appropriate degree of overlapping among the LAs.

\section{System Model And LA Selection Policies}

This section describes the system model and four LA selection policies in details. For the purpose of demonstration, the 1-D overlapping LA configuration is considered. Based on this configuration, in Section III, we describe a random-walk model for MS movement and construct state-transition diagrams to derive the LAU costs.

Figs. 2 and 3 show the 1-D overlapping LA configuration, where each LA covers $N$ cells and is overlapped with each of its adjacent LAs by $K$ cells $(0 \leq K<N)$. We say that the overlapping degree for this LA configuration is $K$. In 


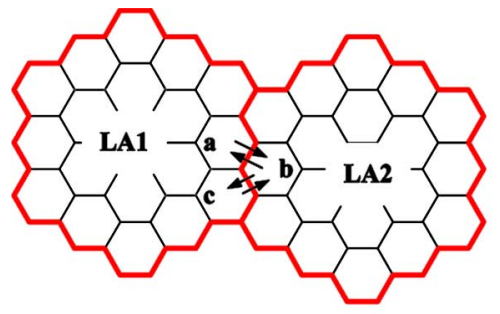

(a)

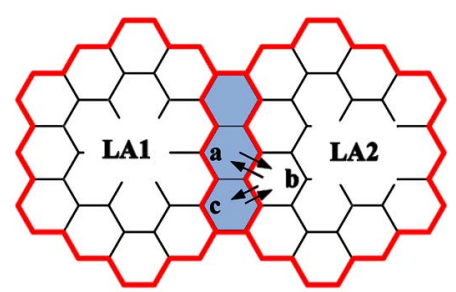

(b)

Fig. 1. Ping-pong effect reduction with overlapping LA configuration. (a) Cellular network with nonoverlapping LAs. (b) Cellular network with overlaping LAs.

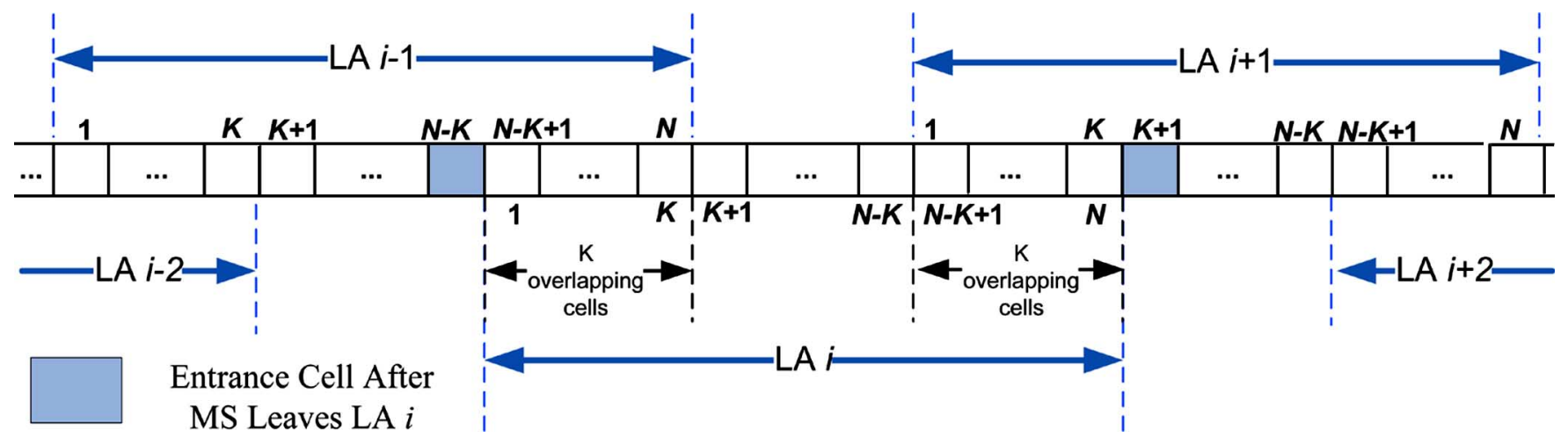

Fig. 2. One-dimensional overlapping LA registration scheme for $0 \leq K<(N / 2)$.

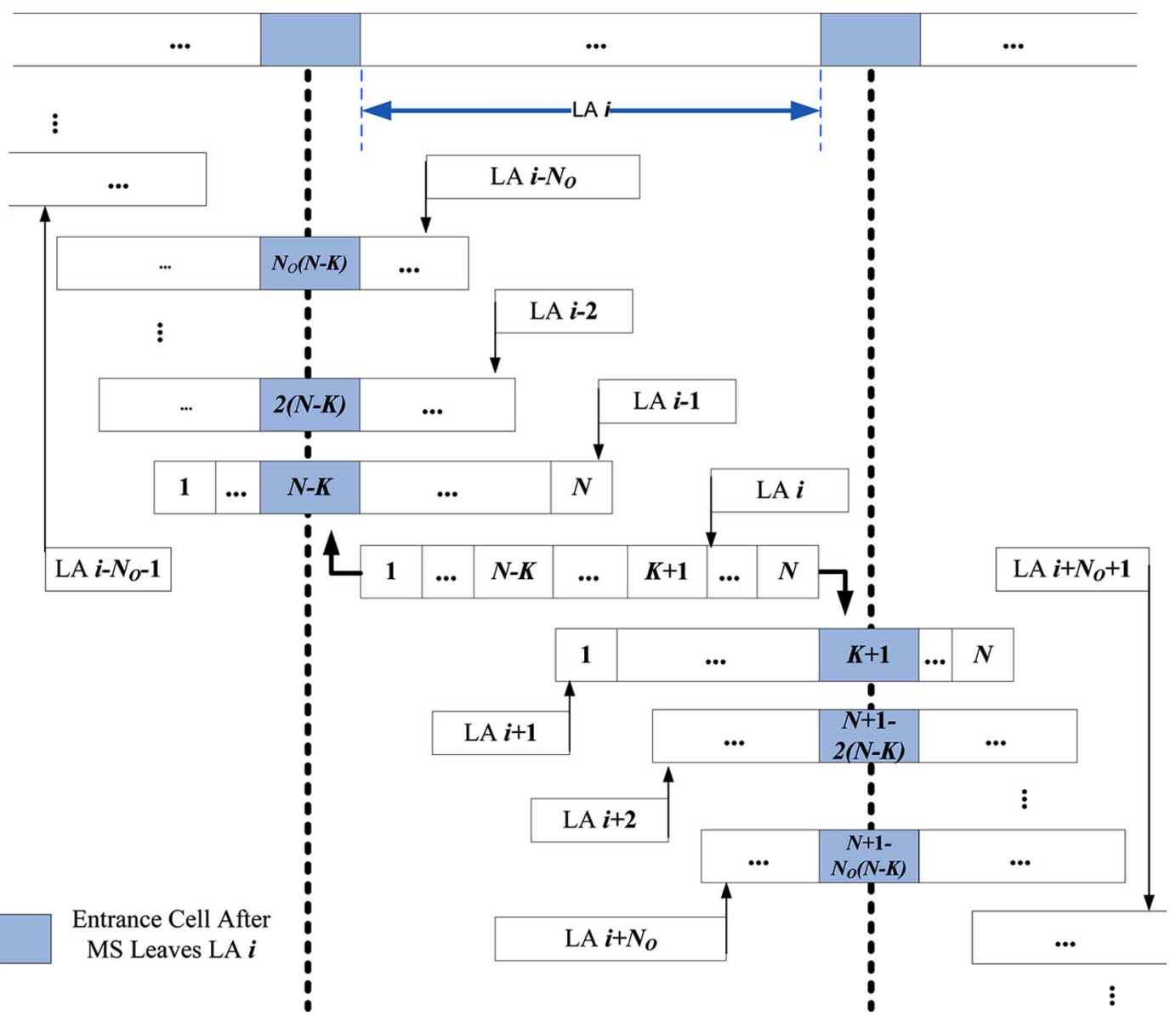

Fig. 3. One-dimensional overlapping LA registration scheme for $(N / 2) \leq K<N$. 


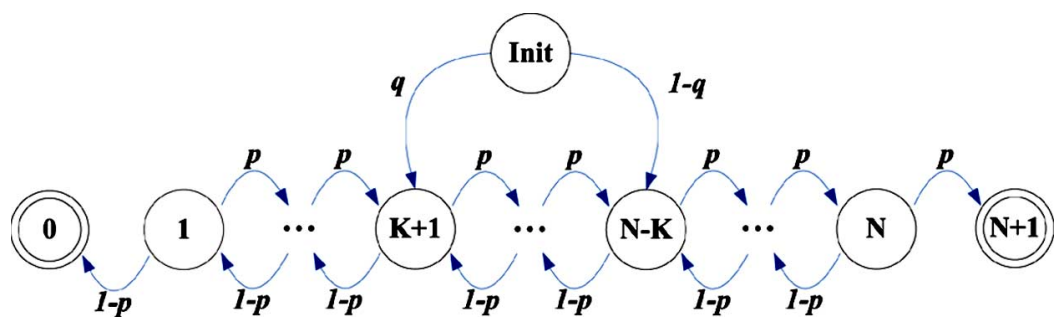

Fig. 4. State-transition diagram for $K$-degree overlapping LA configuration [the LA size is $N$, and $0 \leq K<(N / 2)$ ].

each LA, cells are sequentially labeled from 1 to $N$. An MS moves to the right-hand side neighboring cell with the routing probability $p$ and moves to the left-hand side neighboring cell with probability $1-p$. If the MS moves into a new cell that does not belong to the currently registered LA (i.e., the MS moves out of the current LA), it performs an LAU. This new cell is called the entrance cell to the new LA. If $0 \leq K<(N / 2)$, the entrance cell is covered by only one LA. When the MS moves out of LA $i$ from the right-hand side (the left-hand side), it enters LA $i+1$ (LA $i-1)$. The entrance cell is cell $K+1$ of LA $i+1$ (cell $N-K$ of LA $i-1$ ); see the shadow boxes in Fig. 2. On the other hand, if $(N / 2) \leq K<N$, when an MS leaves the old LA and enters the new cell, this new cell is covered by several LAs adjacent to (or overlapping with) the old LA. As shown in Fig. 3, when the MS leaves LA $i$, the new cell can be the entrance cell for each of $N_{O}$ LAs, where

$$
N_{O}=\left\lceil\frac{K+1}{N-K}\right\rceil \text {. }
$$

That is, if the MS moves out of LA $i$ and enters a right-hand side LA, the entrance cell can be cell $K+1$ of LA $i+1$, cell $N+1-2(N-K)$ of LA $i+2, N+1-3(N-K)$ of LA $i+3, \ldots$, or cell $N+1-N_{O}(N-K)$ of LA $i+N_{O}$ (see the shadow boxes in Fig. 3). Similarly, if the MS moves out of LA $i$ and enters a left-hand side LA, the entrance cell can be cell $N-K$ of LA $i-1$, cell $2(N-K)$ of LA $i-2, \ldots$, or cell $N_{O}(N-K)$ of LA $i-N_{O}$. Since the new cell is covered by more than one LAs for $(N / 2) \leq K<N$, a policy is required to select the new LA at an LAU. In this paper, we investigate four LA selection policies described as follows.

1) In the maximum overlapping (MaxOL) policy [1], [2], [5], after moving out of the current LA $i$ from the righthand side (the left-hand side), the MS will register to the adjacent LA $i+1$ (LA $i-1)$. In this case, the number of cells overlapped between the old and the new LAs is maximal.

2) In the central policy [5], after moving out of the current LA, the MS always registers to the LA whose central cell is closest to the entrance cell.

3) In the random policy, the MS randomly registers to one LA covering the entrance cell.

4) In the minimum overlapping (MinOL) policy, after moving out of LA $i$, the MS chooses the farthest LA of the entrance cell from LA $i$ (i.e., LA $i+N_{O}$ in the righthand side and LA $i-N_{O}$ in the left-hand side in Fig. 3). In this case, the number of cells overlapped between the old and the new LAs is minimal.

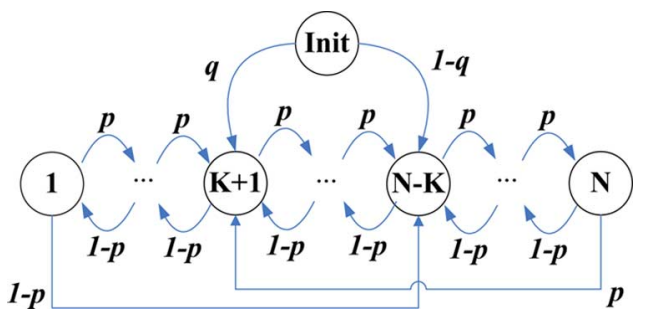

Fig. 5. Modified state-transition diagram for $K$-degree overlapping LA configuration [the LA size is $N$, and $0 \leq K<(N / 2)$ ].

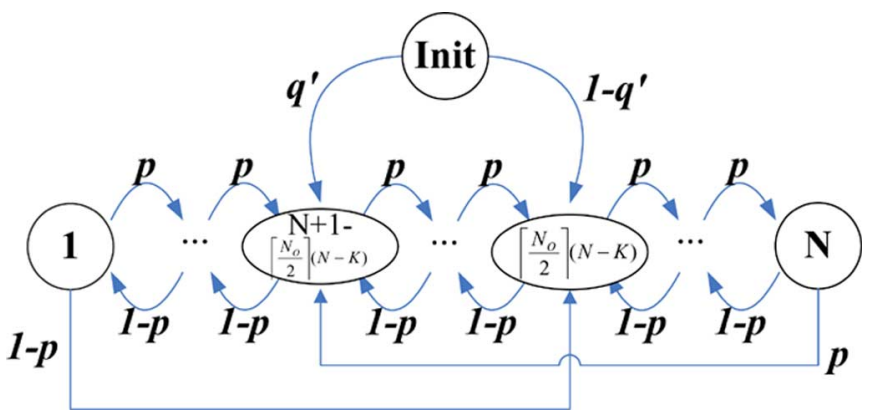

Fig. 6. State-transition diagram for the Central policy $[(N / 2) \leq K<N]$.

Note that for $0 \leq K<(N / 2)$, the aforementioned four policies are the same, i.e., the only LA covering the entrance cell is selected.

\section{ANALYTIC MODEL}

This section proposes an analytic model to study the LAU costs for mobile telecommunications networks with overlapping LAs. Suppose that an MS makes $M$ cell movement before it leaves an LA. For each of the four policies described in Section II, we derive the expected number $E[M]$. It is clear that the larger the $E[M]$ value, the better the performance.

\section{A. Case 1: $0 \leq K<(N / 2)$}

Fig. 4 shows the state-transition diagram for MS cell movement in an LA, where $0 \leq K<(N / 2)$. In this diagram, state Init represents that the MS moves into the LA in the steady state. State $j$ represents that the MS resides in cell $j$ of the LA, where $1 \leq j \leq N$. Two virtual states, 0 and $N+1$, are the absorbing states representing that the MS moves out of the LA from cell 1 and from cell $N$, respectively. For $1 \leq j \leq N$, the MS moves from state $j$ to state $j+1$ with probability $p$, and the MS moves from state $j$ to state $j-1$ with probability $1-p$. As mentioned before, the entrance cell can be cell $K+1$ (cell $N-K$ ) of the new LA when the MS leaves the old LA from 


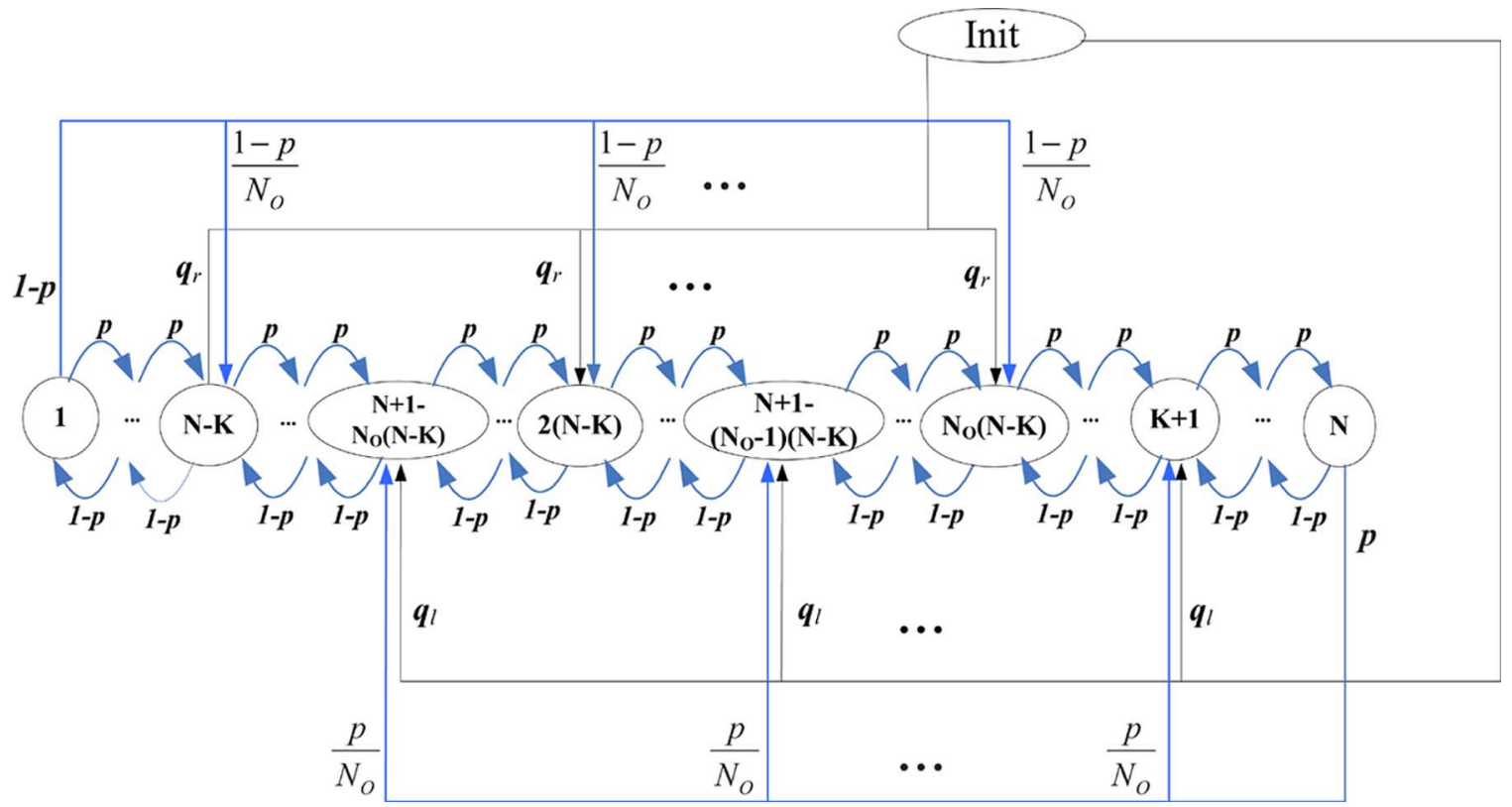

Fig. 7. State-transition diagram for the Random policy $[(N / 2) \leq K<N]$.

the right-hand side (the left-hand side). Let $q$ be the probability that the MS moves from the old LA to the new LA through the entrance cell $K+1$. Then, the MS moves from state Init to state $K+1$ with probability $q$ and to state $N-K$ with probability $1-q$. Note that $q$ is affected by the routing probability $p$, the overlapping degree $K$, and the LA size $N$. We will derive $q$ later.

Starting from the entrance cell $j$, let $N_{j}$ be the number of cell movement before the MS leaves the LA. The expected number $E[M]$ is

$$
E[M]=q E\left[N_{K+1}\right]+(1-q) E\left[N_{N-K}\right] .
$$

We model the MS cell movement as the Gambler's Ruin Problem [10] to solve $E\left[N_{j}\right]$.

Let $\alpha_{j}$ be the probability that starting from cell $j$, the MS will reach state $N+1$ before reaching state 0 (i.e., the MS moves out from the right-hand side). In Fig. 4, we obtain the following recurrence relation for $\alpha_{j}$ :

$$
\alpha_{j}=p \alpha_{j+1}+(1-p) \alpha_{j-1}, \quad \text { for } j=1,2, \ldots, N .
$$

Since $\alpha_{0}=0$ and $\alpha_{N+1}=1$, (3) is solved to yield

$$
\alpha_{j}= \begin{cases}\frac{1-\left(\frac{1-p}{p}\right)^{j}}{1-\left(\frac{1-p}{p}\right)^{N+1,}}, & \text { for } p \neq \frac{1}{2} \\ \frac{j}{N+1,} & \text { for } p=\frac{1}{2} .\end{cases}
$$

Define a random variable $X_{y}$ as follows:

$$
X_{y}= \begin{cases}-1, & \begin{array}{l}
\text { if the MS moves left at } \\
\text { the } y \text { th cell movement }
\end{array} \\
1, & \begin{array}{l}
\text { if the MS moves right at } \\
\text { the } y \text { th cell movement }
\end{array}\end{cases}
$$

Then

$$
N_{j}=\min \left\{n: \sum_{y=1}^{n} X_{y}=-j \quad \text { or } \sum_{y=1}^{n} X_{y}=N+1-j\right\} \text {. }
$$

Note that $E\left[X_{y}\right]=1 \times p+(-1)(1-p)=2 p-1$ and that $N_{j}$ is a stopping time for $X_{y}$ 's. The $E\left[X_{y}\right]$ value can be a positive or a negative number, and the sign of $E\left[X_{y}\right]$ indicates the direction of the MS movement. By using the Wald's equation [10], we have

$$
E\left[\sum_{y=1}^{N_{j}} X_{y}\right]=(2 p-1) E\left[N_{j}\right]
$$

Consider the left-hand side of (5). We have

$$
\sum_{y=1}^{N_{j}} X_{y}= \begin{cases}N+1-j, & \text { with probability } \alpha_{j} \\ -j, & \text { with probability } 1-\alpha_{j}\end{cases}
$$

or

$$
\begin{aligned}
E\left[\sum_{y=1}^{N_{j}} X_{y}\right] & =(N+1-j) \alpha_{j}+(-j)\left(1-\alpha_{j}\right) \\
& =(N+1) \alpha_{j}-j .
\end{aligned}
$$

Substituting (6) into (5) yields

$$
E\left[N_{j}\right]=\frac{(N+1) \alpha_{j}-j}{2 p-1} .
$$

If $p$ approaches (1/2), $E\left[N_{j}\right]$ can be derived by applying the L'Hospital's Rule [11] to (7), which yields

$$
\lim _{p \rightarrow \frac{1}{2}} E\left[N_{j}\right]=(N+1) j-j^{2} .
$$

To derive $q$, Fig. 5 modifies the state diagram in Fig. 4 by removing the absorbing states 0 and $N+1$ and by adding the transitions from state $N$ to state $K+1$ (with probability $p$ ) and from state 1 to state $N-K$ (with probability $1-p$ ). When the MS moves out of the current LA from the right-hand side (the left-hand side), the process moves from state $N$ to state $K+1$ 
(from state 1 to state $N-K$ ). In other words, the MS moves from cell $N$ (cell 1) of the old LA to cell $K+1$ (cell $N-K$ ) of the new LA. In this case, the MS would leave the current LA from the right-hand side boundary and move to cell $K+1$ of new LA with probability $q \alpha_{K+1}$ (i.e., the probability that the MS moves into the entrance cell $K+1$ and then moves out the LA from the right-hand side) plus $(1-q) \alpha_{N-K}$ (i.e., the probability that the MS moves into entrance cell $N-K$ and then moves out the LA from the right-hand side). Since the MS moves from state Init to state $K+1$ with probability $q$, we have the following equation:

$$
q=q \alpha_{K+1}+(1-q) \alpha_{N-K}
$$

or equivalently

$$
q=\frac{\alpha_{N-K}}{1-\alpha_{K+1}+\alpha_{N-K}}
$$

Substituting (4) and (7)-(9) into (2), $E[M]$ is expressed as

$$
E[M]= \begin{cases}\left(\frac{\alpha_{N-K}}{1-\alpha_{K+1}+\alpha_{N-K}}\right) & \\ \times\left[\frac{(N+1) \alpha_{K+1}-K-1}{2 p-1}\right] & \\ +\left(\frac{1-\alpha_{K+1}}{1-\alpha_{K+1}+\alpha_{N-K}}\right) & \\ \times\left[\frac{(N+1) \alpha_{N-K}-N+K}{2 p-1}\right], & \text { for } p \neq \frac{1}{2} \\ (N-K) \times(K+1), & \text { for } p=\frac{1}{2} .\end{cases}
$$

B. Case 2: $(N / 2) \leq K<N$

In this case, an entrance cell is covered by two or more LAs. Therefore, after the MS leaves the old LA, an LA selection policy is required to select the new LA. The $E[M]$ values for the four policies are derived as follows.

1) MaxOL: The MS always chooses a new LA with maximum overlapping with the old LA. When the MS moves out of LA $i$ from the right-hand side (the left-hand side), it registers to LA $i+1$ (LA $i-1)$. Clearly, the LA selected in this policy is the same as that selected in case $0 \leq K<(N / 2)$ described in Section III-A. Therefore, the expected number of MS cell movement in an LA is expressed in (10).

2) Central: After moving out of LA $i$, the MS always registers to the LA whose central cell is closest to the entrance cell. The selected LA is the $\left[N_{O} / 2\right\rceil$ th LA away from LA $i$. That is, the entrance cell is cell $N+1-$ $\left\lceil N_{O} / 2\right\rceil(N-K)$ of LA $i+\left\lceil N_{O} / 2\right\rceil$ on the right-hand side or cell $\left\lceil N_{O} / 2\right\rceil(N-K)$ of LA $i-\left\lceil N_{O} / 2\right\rceil$ on the left-hand side. The state-transition diagram for the Central policy is shown in Fig. 6, where the MS moves from state Init to state $N+1-\left\lceil N_{O} / 2\right\rceil(N-K)$ with probability $q^{\prime}$ and to state $\left\lceil N_{O} / 2\right\rceil(N-K)$ with probability $1-q^{\prime}$. Following the derivation in Section III-A, we have

$$
q^{\prime}=\frac{\alpha\left\lceil\frac{N_{O}}{2}\right\rceil(N-K)}{1-\alpha_{N+1-\left\lceil\frac{N_{O}}{2}\right\rceil(N-K)}+\alpha\left\lceil\frac{N_{O}}{2}\right\rceil(N-K)}
$$

TABLE I

ANALYTIC AND Simulation Results $(N=15)$

\begin{tabular}{|c|c|c|c|c|}
\hline K & 0 & 4 & 8 & 12 \\
\hline \multicolumn{5}{|c|}{$p=0.5$} \\
\hline E[M] (Analytic) & 15 & 55 & 63 & 39 \\
\hline E[M] (Simulation) & 15.022 & 54.979 & 62.913 & 38.845 \\
\hline Error & $-0.152 \%$ & $0.037 \%$ & $0.136 \%$ & $0.396 \%$ \\
\hline \multicolumn{5}{|c|}{$p=0.7$} \\
\hline E[M] (Analytic) & 15 & 26.716 & 17.482 & 7.499 \\
\hline E[M] (Simulation) & 15.032 & 26.622 & 17.458 & 7.501 \\
\hline Error & $-0.214 \%$ & $0.351 \%$ & $0.141 \%$ & $-0.023 \%$ \\
\hline
\end{tabular}

(a) The MaxOL Policy

\begin{tabular}{|c|c|c|c|c|}
\hline K & 0 & 4 & 8 & 12 \\
\hline \multicolumn{5}{|c|}{$p=0.5$} \\
\hline E[M] (Analytic) & 15 & 55 & 63 & 63 \\
\hline E[M] (Simulation) & 14.987 & 55.060 & 62.925 & 62.927 \\
\hline Error & $0.084 \%$ & $-0.109 \%$ & $0.118 \%$ & $0.115 \%$ \\
\hline \multicolumn{5}{|c|}{$p=0.7$} \\
\hline E[M] (Analytic) & 15 & 26.716 & 17.482 & 22.380 \\
\hline E[M] (Simulation) & 15.032 & 26.622 & 17.465 & 22.330 \\
\hline Error & $-0.214 \%$ & $0.351 \%$ & $0.100 \%$ & $0.223 \%$ \\
\hline
\end{tabular}

(b) The Central Policy

\begin{tabular}{|c|c|c|c|c|}
\hline K & 0 & 4 & 8 & 12 \\
\hline \multicolumn{5}{|c|}{$p=0.5$} \\
\hline E[M] (Analytic) & 15 & 55 & 45.5 & 45 \\
\hline E[M] (Simulation) & 15.003 & 54.899 & 45.129 & 44.833 \\
\hline Error & $-0.020 \%$ & $0.181 \%$ & $0.814 \%$ & $0.370 \%$ \\
\hline \multicolumn{5}{|c|}{$p=0.7$} \\
\hline E[M] (Analytic) & 15 & 26.716 & 21.818 & 18.608 \\
\hline E[M] (Simulation) & 15.025 & 26.738 & 21.992 & 18.579 \\
\hline Error & $-0.169 \%$ & $-0.083 \%$ & $-0.798 \%$ & $0.157 \%$ \\
\hline
\end{tabular}

(c) The Random Policy

\begin{tabular}{|c|c|c|c|c|}
\hline K & 0 & 4 & 8 & 12 \\
\hline \multicolumn{5}{|c|}{$p=0.5$} \\
\hline E[M] (Analytic) & 15 & 55 & 28 & 15 \\
\hline E[M] (Simulation) & 15.003 & 54.899 & 27.969 & 14.995 \\
\hline Error & $-0.020 \%$ & $0.181 \%$ & $0.107 \%$ & $0.031 \%$ \\
\hline \multicolumn{5}{|c|}{$p=0.7$} \\
\hline E[M] (Analytic) & 15 & 26.716 & 24.137 & 15 \\
\hline E[M] (Simulation) & 15.025 & 26.738 & 24.136 & 15.008 \\
\hline Error & $-0.169 \%$ & $-0.083 \%$ & $0.007 \%$ & $-0.056 \%$ \\
\hline
\end{tabular}

(d) The MinOL Policy

and the expected number is in (11), shown at the bottom of the next page.

3) Random: The MS randomly registers to one LA covering the entrance cell. Let $q_{l}\left(q_{r}\right)$ be the probability that the MS moves from a left-hand side LA (right-hand side LA) to the new LA through the entrance cell. The state-transition diagram for the Random policy is shown in Fig. 7, where the MS moves from state Init to state $N+1-$ $m(N-K)$ with probability $q_{l}$ and to state $m(N-K)$ with probability $q_{r}$, where $1 \leq m \leq N_{O}$. In Fig. 7, $E[M]$ is expressed as

$$
\begin{aligned}
E[M]=q_{l}\left\{\sum_{m=1}^{N_{O}} E\left[N_{N+1-m(N-K)}\right]\right\} & \\
& +q_{r}\left\{\sum_{m=1}^{N_{O}} E\left[N_{m(N-K)}\right]\right\} .
\end{aligned}
$$

Since the entrance cell is covered by $N_{O}$ LAs, when the MS leaves the old LA, the process moves from 


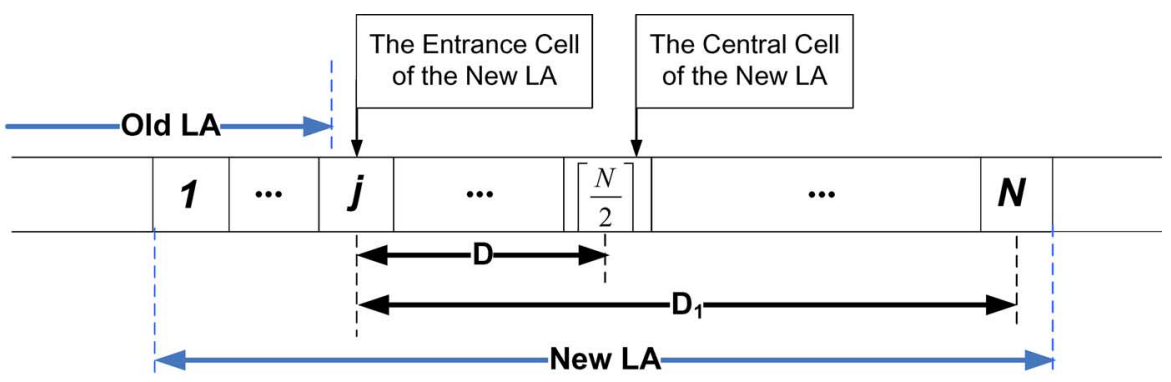

(a) $\mathbf{j} \leq\left\lceil\frac{N}{2}\right\rceil$

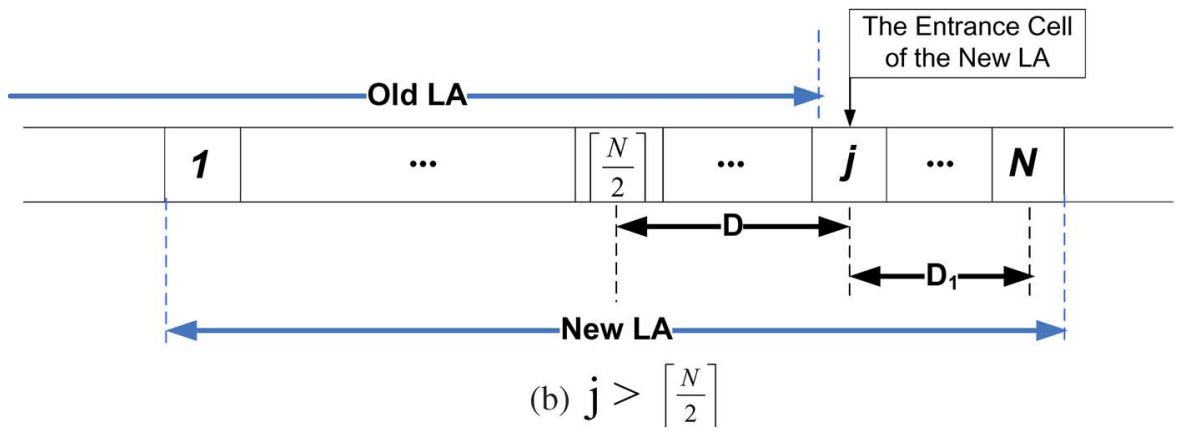

Fig. 8. Distances $D$ and $D_{1}$ in the new LA.

state $N$ (state 1) to state $N+1-m(N-K)$ (state $m(N-K))$ with probability $\left(p / N_{O}\right)\left((1-p) / N_{O}\right)$, where $1 \leq m \leq N_{O}$ (see Fig. 7). From the balance equations for the states previously described, probabilities $q_{r}$ and $q_{l}$ can be computed. Then, $E[M]$ for the Random policy can be derived by substituting these probabilities into (12). The details are omitted.

4) MinOL: After leaving LA $i$, the MS chooses the farthest LA of the entrance cell from LA $i$. The entrance cell is cell $N+1-N_{O}(N-K)$ of LA $i+N_{O}$ on the righthand side or cell $N_{O}(N-K)$ of LA $i-N_{O}$ on the lefthand side. Derivation of $E[M]$ for this policy is similar to that for case $0 \leq K<(N / 2)$, and the details are omitted.

Note that our analytic results have been validated against the simulation experiments (see Table I). The errors between the analytic and simulation models are under $1 \%$.

\section{Performance Evaluation}

This section studies the performance of the LA selection policies (i.e., MaxOL, Central, Random, and MinOL). Specifically, we investigate the expected number $E[M]$ of cell movement in an LA before an MS leaves the LA. It is clear that the larger the $E[M]$ value, the better the performance. In our numerical examples, we show the results for LA size $N=15$. The results for other $N$ values are similar and are omitted.

We first note that due to the symmetric cell structure in each LA, the effect for $p$ is identical to that for $1-p$. Therefore, it suffices to consider $0.5 \leq p \leq 1$. Denote $D$ as the distance (the number of cells) between the entrance cell (cell $j$ in Fig. 8) and the central cell of the new LA (cell $\lceil N / 2\rceil$ in Fig. 8).

Denote $D_{1}$ as the distance between the entrance cell and the new LA's right-hand side (left-hand side) boundary cell if the MS enters the new LA from the left-hand side (right-hand side) of the LA (cell $N$ in Fig. 8). Note that $E[M]$ is affected by the ping-pong effect and moving-to-one-direction effect. Besides

$$
\begin{aligned}
& E[M]=q^{\prime} E\left[N_{N+1-\left\lceil\frac{N_{O}}{2}\right\rceil(N-K)}\right]+\left(1-q^{\prime}\right) E\left[N_{\left\lceil\frac{N_{O}}{2}\right\rceil(N-K)}\right]
\end{aligned}
$$

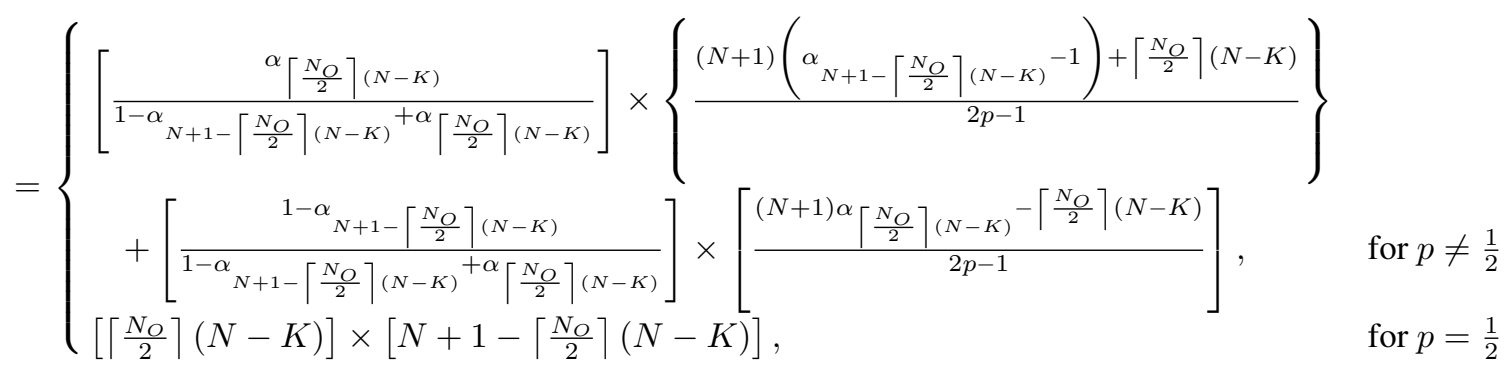


TABLE II

Distance $D$ Between the Entrance Cell and the Central Cell of the New LA $(N=15)$

\begin{tabular}{|l|c|c|c|c|c|c|c|c|c|c|c|c|c|c|c|}
\hline \multicolumn{1}{|c|}{$\mathrm{K}$} & 0 & 1 & 2 & 3 & 4 & 5 & 6 & 7 & 8 & 9 & 10 & 11 & 12 & 13 & 14 \\
\hline \hline MaxOL & 7 & 6 & 5 & 4 & 3 & 2 & 1 & 0 & 1 & 2 & 3 & 4 & 5 & 6 & 7 \\
\hline Cental & 7 & 6 & 5 & 4 & 3 & 2 & 1 & 0 & 1 & 2 & 2 & 0 & 1 & 0 & 0 \\
\hline Random & 7 & 6 & 5 & 4 & 3 & 2 & 1 & 0 & 3.5 & 3 & 4 & 2.7 & 3.8 & 3.4 & 3.7 \\
\hline MinOL & 7 & 6 & 5 & 4 & 3 & 2 & 1 & 0 & 6 & 4 & 7 & 4 & 7 & 6 & 7 \\
\hline
\end{tabular}

TABLE III

Distance $D_{1}(N=15)$

\begin{tabular}{|c|c|c|c|c|c|c|c|c|c|c|c|c|c|c|c|}
\hline $\mathrm{K}$ & 0 & 1 & 2 & 3 & 4 & 5 & 6 & 7 & 8 & 9 & 10 & 11 & 12 & 13 & 14 \\
\hline \hline MaxOL & 14 & 13 & 12 & 11 & 10 & 9 & 8 & 7 & 6 & 5 & 4 & 3 & 2 & 1 & 0 \\
\hline Cental & 14 & 13 & 12 & 11 & 10 & 9 & 8 & 7 & 6 & 5 & 9 & 7 & 8 & 7 & 7 \\
\hline Random & 14 & 13 & 12 & 11 & 10 & 9 & 8 & 7 & 9.5 & 8 & 9 & 7 & 8 & 7 & 7 \\
\hline MinOL & 14 & 13 & 12 & 11 & 10 & 9 & 8 & 7 & 13 & 11 & 14 & 11 & 14 & 13 & 14 \\
\hline
\end{tabular}

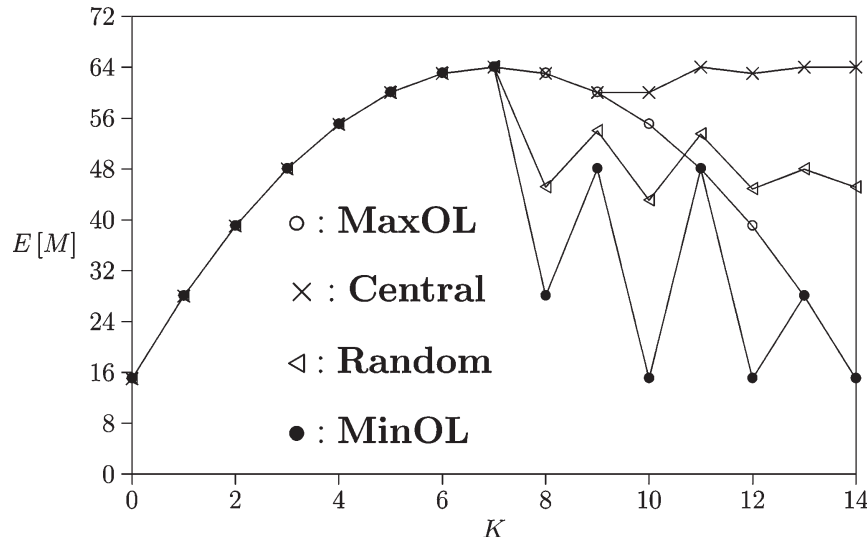

Fig. 9. Effects of $K$ on $E[M]$ for $p=0.5$ and $N=15$.

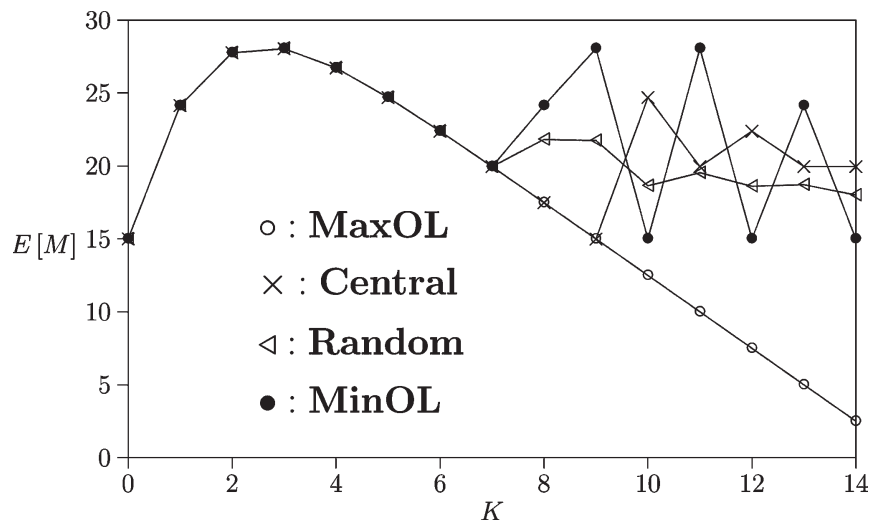

Fig. 10. Effects of $K$ on $E[M]$ for $p=0.7$ and $N=15$.

$p$, these effects are determined by $D$ and $D_{1}$. The smaller the $D$ value, the less significant the ping-pong effect. The larger the $D_{1}$ value, the less significant the moving-to-one-direction effect. Tables II and III list $D$ and $D_{1}$ as functions of $K$ for $N=15$. These $D$ and $D_{1}$ values are computed from the analytic model and are validated by the simulation experiments.

Figs. 9-12 show $E[M]$ as a function of $K$ and $p$, where $N=15$. Consider $0.5 \leq p \leq 1$. When $p$ is small, the pingpong effect is significant, and $E[M]$ is likely to increase as $D$ decreases. When $p=0.5, E[M]$ is a decreasing function of $D$ (see Fig. 9 and Table II). When $p=1$, the MS always moves in one direction. Therefore, $E[M]$ increases as $D_{1}$ increases (see

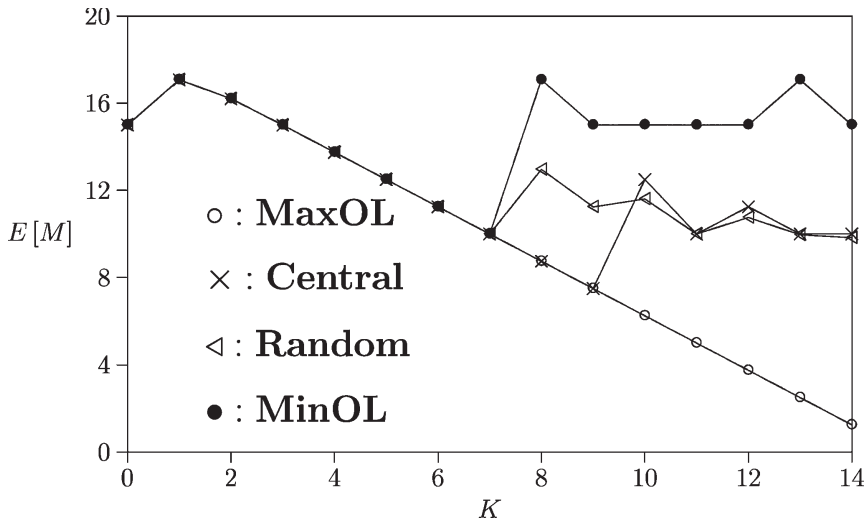

Fig. 11. Effects of $K$ on $E[M]$ for $p=0.9$ and $N=15$.

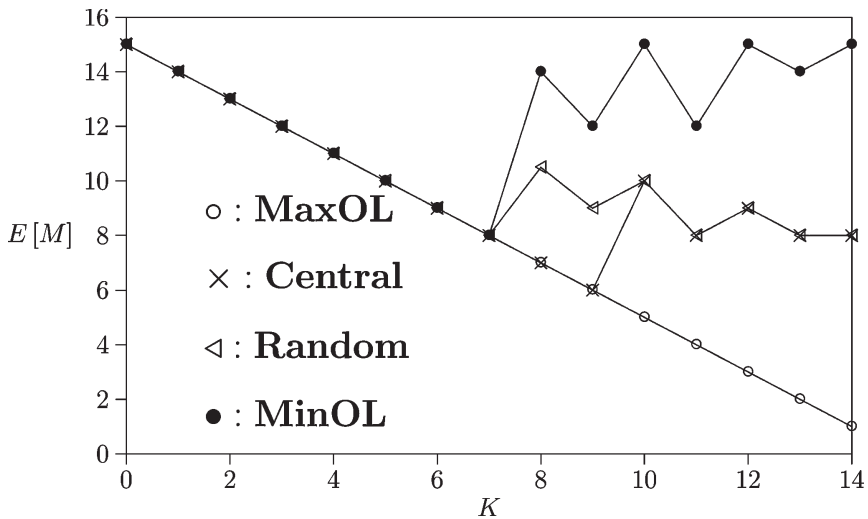

Fig. 12. Effects of $K$ on $E[M]$ for $p=1.0$ and $N=15$.

Fig. 12 and Table III). When $p$ increases, the ping-pong effect (impact of $D$ ) becomes insignificant, and the moving-to-onedirection effect (impact of $D_{1}$ ) becomes significant. Considering the MaxOL policy, $E[M]$ increases and then decreases as $K$ increases. For $p=0.5,0.7,0.9,1$, the maximal $E[M]$ values occur when $K=7,3,1,0$, respectively. For a maximal $E[M]$ value, the corresponding $K$ value is called the best overlapping degree. As $p$ increases, the impact of $D_{1}$ becomes more significant, and the best overlapping degree decreases.

In Figs. 9-12, we observe that when $0 \leq K<(N / 2)$, all four policies result in the same performance. When $(N / 2) \leq$ $K<N$, the Central policy makes sense for $p=0.5$, while the MinOL policy is more appropriate for $p=1.0$. For all policies, these figures show an apparent result that for the same $K$ value, $E[M]$ decreases as $p$ increases. By differentiating the analytic equations [e.g., (10) and (11)], we can formally prove that the best overlapping degree always occurs when $0 \leq K<(N / 2)$. That is, in a 1-D overlapping LA configuration, it suffices to consider the configurations for $K<(N / 2)$.

\section{CONClusion}

This paper studied four LA selection policies for overlapping LA configuration: MaxOL, Central, Random, and MinOL policies. We proposed an analytic model to investigate the $E[M]$ performance of these policies for the 1-D overlapping LA configuration. The analytic results were validated against the simulation experiments. 
Our study for the 1-D overlapping LA configuration is more general than the previous studies [1], [2], [5]. For specific scenarios in the previous studies, their results are consistent with ours (which validates that our results are correct). The major difference between our model and those in the previous studies is the setup for the routing probability $p$. All previous studies assumed that at each movement, the MS moves to any of its neighboring cells with the same routing probability. In our study, we assume that the MS moves to each of its neighboring cells with different probabilities (i.e., $0<p \leq 1.0$ ). Furthermore, the overlapping degree $K$ in our study can be arbitrary (i.e., $0 \leq K<N)$. Previous studies made a restrictive assumption, where $0 \leq K<(N / 2)$. We note that there is a typo in [5, eq. (11)]. Specifically, the $(2 d-w+1)$ term in the righthand side of the equation should be rewritten as $(2 d-w-1)$.

Our study indicates the following results.

1) It suffices to consider the configurations for $0 \leq K<$ $(N / 2)$, and all four policies result in the same performance in these configurations. When $(N / 2) \leq K<N$, the Central policy makes sense for $p=0.5$, while the MinOL policy is more appropriate for $p=1.0$.

2) When the routing probability $p \in[0.5,1]$, the $E[M]$ value decreases as $p$ increases. When $p=1$ (i.e., the mobile telecommunications network is deployed in the highway), the $E[M]$ performance for $K=0$ is always better than that for $K>0$.

From the numerical comparison of the four LA selection policies, our study indicates that in practical scenarios, the best overlapping degree occurs for $0 \leq K<(N / 2)$, and it suffices to consider the MaxOL policy. This important result has not been reported in the literature. In the future, we will extend our 1-D model for a 2-D LA layout.

\section{ACKNOWLEDGMENT}

The authors would like to thank the anonymous reviewers for their valuable comments that have significantly improved the quality of this paper.

\section{REFERENCES}

[1] K.-H. Chiang and N. Shenoy, "Performance of an overlapped macro-cell based location area scheme," in Proc. ICC, May 2003, vol. 1, pp. 475-481.

[2] K.-H. Chiang and N. Shenoy, "A 2-D random-walk mobility model for location-management studies in wireless networks," IEEE Trans. Veh. Technol., vol. 53, no. 2, pp. 413-424, Mar. 2004.

[3] G. Fan and J. Zhang, "A multi-layer location management scheme that bridges the best static scheme and the best dynamic scheme," in Proc. Int. Conf. MDM, 2004, pp. 125-132.

[4] G. Fan, I. Stojmenovic, and J. Zhang, "A triple layer location management strategy for wireless cellular networks," in Proc. IEEE Int. Conf. Comput. Commun. Netw., 2002, pp. 489-492.

[5] D. Gu and S. S. Rappaport, "Mobile user registration in cellular system with overlapping location areas," in Proc. IEEE Veh. Technol. Conf., May 1999, vol. 1, pp. 802-806.

[6] Institute of Electrical and Electronics Engineers, IEEE Standard for Local and Metropolitan Area Networks-Part 16: Air Interface for Fixed and Mobile Broadband Wireless Access Systems-Amendment 2: Physical and Medium Access Control Layers for Combined Fixed and Mobile Operation in Licensed Bands and Corrigendum 1, IEEE Std. 802.16e$2005,2005$.

[7] Y.-B. Lin and I. Chlamtac, Wireless and Mobile Network Architectures. Hoboken, NJ: Wiley, 2001.
[8] Y.-B. Lin and A.-C. Pang, Wireless and Mobile All-IP Networks. Hoboken, NJ: Wiley, 2005.

[9] S. Okasaka, S. Onoe, S. Yasuda, and A. Maebara, "A new location updating method for digital cellular systems," in Proc. 41st Annи. Conf. VTS, May 1991, pp. 345-350.

[10] S. M. Ross, Stochastic Processes, 2nd ed. Hoboken, NJ: Wiley, 1996.

[11] G. B. Thomas and R. L. Finney, Calculus and Analytic Geometry, 9th ed. Reading, MA: Addison-Wesley, 1996.

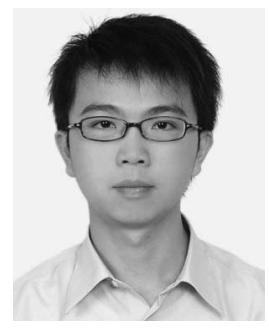

Shun-Ren Yang received the B.S. and M.S. degrees in computer science and information engineering and the Ph.D. degree from the National Chiao Tung University, Hsinchu, Taiwan, R.O.C., in 1998, 1999, and 2004, respectively.

From April 1, 2004 to July 31, 2004, he was a Research Assistant with the Department of Information Engineering, The Chinese University of Hong Kong, Sha Tin, Hong Kong. Since August 2004, he has been with the Department of Computer Science and Institute of Communications Engineering, National Tsing Hua University, Hsinchu, where he is currently an Assistant Professor. His current research interests include the design and analysis of personal communications services networks, computer telephony integration, mobile computing, and performance modeling.

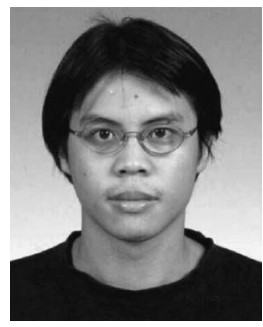

Yung-Chun Lin received the B.S. and M.S. degrees in computer science and information engineering from the National Chiao Tung University, Hsinchu, Taiwan, R.O.C., in 2001 and 2003, respectively, where he is currently working toward the Ph.D. degree in computer science with the Department of Computer Science.

His research interests include the design and analysis of a personal communications services network, the cellular protocols (UMTS/GPRS/GSM), and mobile computing.

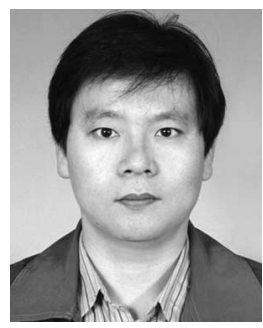

Yi-Bing Lin (M'95-SM'95-F'03) He is currently a Chair Professor and Dean of the Department of Computer Science, National Chiao Tung University, Hsinchu, Taiwan, R.O.C. He is also with the Institute of Information Science, Academia Sinica, Taipei, Taiwan. He has published over 200 journal articles and more than 200 conference papers. He is the coauthor of the books Wireless and Mobile Network Architecture (with I. Chlamtac, Wiley, 2001) and Wireless and Mobile All-IP Networks (with A. -C. Pang, Wiley, 2005). His current research interests include mobile computing and cellular telecommunications services.

Dr. Lin is a Fellow of Association for Computing Machinery, the American Association for the Advancement of Science, and the Institution of Electrical Engineers. 